\section{Quando a HbA1c não é suficiente: um caso de Hemoglobina de Baltimore}

\author{
When HbA1c is not enough: a case of Baltimore Hemoglobin \\ Cuando la HbA1c no es suficiente: un caso de Hemoglobina de \\ Baltimore
}

\section{Resumo}

A hemoglobina $\mathrm{A} 1 \mathrm{c}(\mathrm{HbA} 1 \mathrm{c})$ é o gold standard para monitorização da diabetes mellitus. A $\mathrm{HbA1c}$ pode estar falsamente diminuída ou aumentada em algumas situações clínicas, como hemoglobinopatias, não traduzindo adequadamente o controle glicêmico. Apresenta-se aqui o caso de um doente com valor de $\mathrm{HbA} 1 \mathrm{c}$ incompatível com os registros de glicemia capilar, devido à presença de hemoglobina $\mathrm{N}$-Baltimore. $\mathrm{O}$ caso apresentando é relevante porque, apesar de assintomática, a presença desta hemoglobinopatia conduz a uma diabetes falsamente encarada como controlada, se utilizados os métodos de determinação de $\mathrm{HbA1c}$ habituais.

Palavras-chave: Diabetes Mellitus; Hemoglobina A Glicada; Hemoglobinas Anormais

\begin{abstract}
The haemoglobin $\mathrm{A} 1 \mathrm{c}(\mathrm{HbA1c})$ test is the gold standard in monitoring diabetes mellitus. $\mathrm{HbA1c}$ can be falsely decreased or increased in some particular scenarios, as in hemoglobinopathies, inadequately reporting the glycemic control. Here we present the case of a patient with a $\mathrm{HbA} 1 \mathrm{c}$ value incompatible with self-monitoring blood glucose levels, due to the presence of hemoglobin N-Baltimore. This case is relevant because, in spite of being asymptomatic, the presence of this type of hemoglobin leads to an incorrect appearance of glycemic control, if standard methods for $\mathrm{HbA} 1 \mathrm{c}$ determination are used.
\end{abstract}

Keywords: Diabetes Mellitus; Glycated Hemoglobin A; Hemoglobins, Abnormal

\section{Resumen}

La hemoglobina $\mathrm{A} 1 \mathrm{c}(\mathrm{HbA} 1 \mathrm{c})$ es el estándar de oro para la monitorización de la diabetes mellitus. La HbA1c puede estar falsamente disminuida o aumentada en algunas situaciones clínicas, como hemoglobinopatías, no traduciendo adecuadamente el control glucémico. Se presenta aquí el caso de un paciente con valor de $\mathrm{HbA} 1 \mathrm{c}$ incompatible con los registros de glucemia capilar, debido a la presencia de hemoglobina N-Baltimore. El caso que presenta es relevante porque, a pesar de asintomática, la presencia de esta hemoglobinopatía conduce a una diabetes falsamente encarada como controlada, si se utilizan los métodos de determinación de HbA1c habituales.

Palabras clave: Diabetes Mellitus; Hemoglobina A Glucada; Hemoglobinas Anormales

Como citar: Costa AS, Ramalho A, Lima M, Bitoque A. Quando a HbA1c não é suficiente: um caso de Hemoglobina de Baltimore. Rev Bras Med Fam Comunidade. 2018;13(40):1-6. http://dx.doi.org/10.5712/rbmfc13(40)1746
André Silva Costa
Andreia Ramalho $^{\mathrm{b}}$
Marina Lima
Anabela Bitoque $^{\mathrm{a}}$

a Unidade de Saúde Familiar Poente. Portugal. andrefsc88@gmail.com (Autor correspondente); anabelabitoque@gmail.com

b Unidade de Saúde Familiar Sobreda. Portugal. and_ramalho@hotmail.com

c Unidade Saúde Familiar Cova da Piedade. Portugal. marina_aml@ hotmail.com

Fonte de financiamento: declaram não haver. Parecer CEP: não se aplica. Conflito de interesses: declaram não haver. Procedência e revisão por pares: revisado por pares. Recebido em: 05/05/2018. Aprovado em: 14/09/2018. 


\section{Introdução}

A hemoglobina glicada $(\mathrm{HbA} 1 \mathrm{c})$ reflete a glicemia média dos últimos 2 a 3 meses, correspondendo à semivida dos eritrócitos, sendo o método regularmente utilizado para monitorizar o controle metabólico na diabetes. Níveis de $\mathrm{HbA} 1 \mathrm{c}$ acima de $7 \%$ estão associados a um risco progressivamente maior de complicações da diabetes. O valor alvo para cada indivíduo pode ser maior ou menor, dependendo das suas características clínicas particulares. ${ }^{1}$

A HbA1c pode estar falsamente diminuída em certas situações clínicas, não traduzindo adequadamente o controle glicêmico. ${ }^{2,3}$ São exemplos destas situações hemoglobinopatias e anemia hemolítica, bem como outras condições que afetem o tempo de vida do eritrócito ou a ligação entre a hemoglobina e a glicose (gravidez, hemorragia, terapêutica de reposição com ferro e vitamina B12, doença renal crônica - DRC). ${ }^{1-3}$ Estudos epidemiológicos relataram diferenças étnicas na $\mathrm{HbA} 1 \mathrm{c}$, tendo os afro-americanos, em média, valores de $\mathrm{HbA} 1 \mathrm{c}$ superiores aos europeus. ${ }^{4}$

Mais de 1300 variantes de $\mathrm{Hb}$ foram descritas até à data. ${ }^{5} \mathrm{Hemoglobinas} \mathrm{variantes} \mathrm{possuem} \mathrm{estrutura}$ química diferente da hemoglobina normal correspondente, resultante de mutações em uma ou mais bases azotadas, com troca de aminoácidos nas cadeias alfa, beta, delta ou gama. ${ }^{6}$ Enquanto certas variantes são comuns e associadas a quadros clínicos clássicos, como a anemia falciforme, outras são relativamente incomuns e clinicamente silenciosas. ${ }^{2,5}$

Algumas destas hemoglobinopatias podem afetar a determinação da HbA1c por HPLC (High performance liquid chromatography), ${ }^{2}$ como é o caso da variante $\mathrm{Hb}$ N-Baltimore ( $\beta 95$ Lys $\left.\rightarrow \mathrm{Glu}\right) .{ }^{1}$ Por não apresentarem sintomas clínicos, os portadores desta hemoglobina são detectados apenas por estudos populacionais ou quando a $\mathrm{Hb} \mathrm{N}$ estiver associada a formas talassêmicas ou a outras variantes da hemoglobina, ${ }^{6}$ não existindo dados relativamente à sua prevalência.

Apresenta-se aqui o caso de um paciente diabético com determinações de $\mathrm{HbA} 1 \mathrm{c}$ não compatíveis com o registo de glicemias capilares, devido à presença de $\mathrm{Hb}$ N-Baltimore, com importantes implicações para a sua monitorização e seguimento.

\section{Detalhamento do caso}

J.A.D.R., 63 anos, raça caucasiana, taxista, inserido numa família nuclear, no estádio VI do Ciclo da vida de Duvall (família com adulto jovem a sair de casa). Apresentava como antecedentes pessoais diabetes mellitus tipo 2, Hipertensão Arterial Essencial grau 1 (controlada, sem lesão de órgão alvo) e obesidade (Índice de Massa Corporal - IMC - 30kg/m²). Estava medicado com Losartan 100mg, 1 comprimido de manhã, e Metformina 700mg, 1 comprimido ao almoço. Negava consumos tabagistas, etílicos ou toxicofílicos. Sem história familiar de relevo.

O diagnóstico de diabetes mellitus foi realizado aos 59 anos (4 anos de evolução da doença) após 2 valores de glicemia em jejum $\geq 126 \mathrm{mg} / \mathrm{dL}$ (nomeadamente, 140 e $142 \mathrm{mg} / \mathrm{dL}$, com intervalo de 6 semanas).

O utente recorreu à consulta de vigilância de diabetes com o seu Médico de Família para a qual trazia resultados de análises, das quais se destacava HbA1c (HPLC - troca catiônica, Menarini ${ }^{\circledR}$ Arkray Adams A1c HA818O) de 5,4\%. O paciente encontrava-se assintomático, negando poliúria, polidipsia ou perda de 
peso. Negava igualmente sintomas de hipoglicemia. Trazia registros de autocontroles de glicemias capilares dos últimos 3 dias com valores em jejum entre 188-291 mg/dL e pós-prandiais entre $240-297 \mathrm{mg} / \mathrm{dL}$.

Ao exame objetivo, apresentava IMC de $30 \mathrm{~kg} / \mathrm{m}^{2}$. O seu pulso era regular a $76 \mathrm{bpm}$ com uma tensão arterial de 138/86mmHg. A auscultação cardiopulmonar não revelou alterações de relevo. Na avaliação do risco de pé diabético tinha um risco baixo (2015 International Working Group on the Diabetic Foot). Apresentava acuidade visual preservada, com rastreio de retinopatia diabética por retinografia, no ano anterior, com resultado negativo.

Verificou-se que o valor de $\mathrm{HbA} 1 \mathrm{c}$ que o paciente apresentava não era compatível com os registros das suas glicemias capilares. A primeira questão a ser abordada com o paciente foi a técnica de medição da glicemia capilar, na qual verificamos não existirem erros grosseiros que interferissem com os valores obtidos. Considerando que existem algumas situações clínicas (hemoglobinopatias, anemia hemolítica, $\mathrm{DRC}$ ) que podem interferir com os valores de $\mathrm{HbA1c}$, foi pedido, na consulta de Medicina Geral e Familiar, estudo analítico para exclusão destas causas.

Duas semanas depois, o utente traz os resultados das análises solicitadas (Tabela 1), destacando-se a eletroforese das hemoglobinas, com fração de $54,4 \%$ de $\mathrm{Hb}$ Variante, compatível com hemoglobina N-Baltimore. É então pedida nova HbA1c pelo método HPLC conjugado com a afinidade ao boronato (Menarini ${ }^{\circledR}$ Hb912O Premier), obtendo-se um valor de 8,4\% e fructosamina de $604 \mu \mathrm{mol} / \mathrm{L}(\mathrm{IR}<285)$.

Tabela 1. Resultados analíticos pedidos após detectar-se incongruência entre as glicemias capilares e a HbA1c.

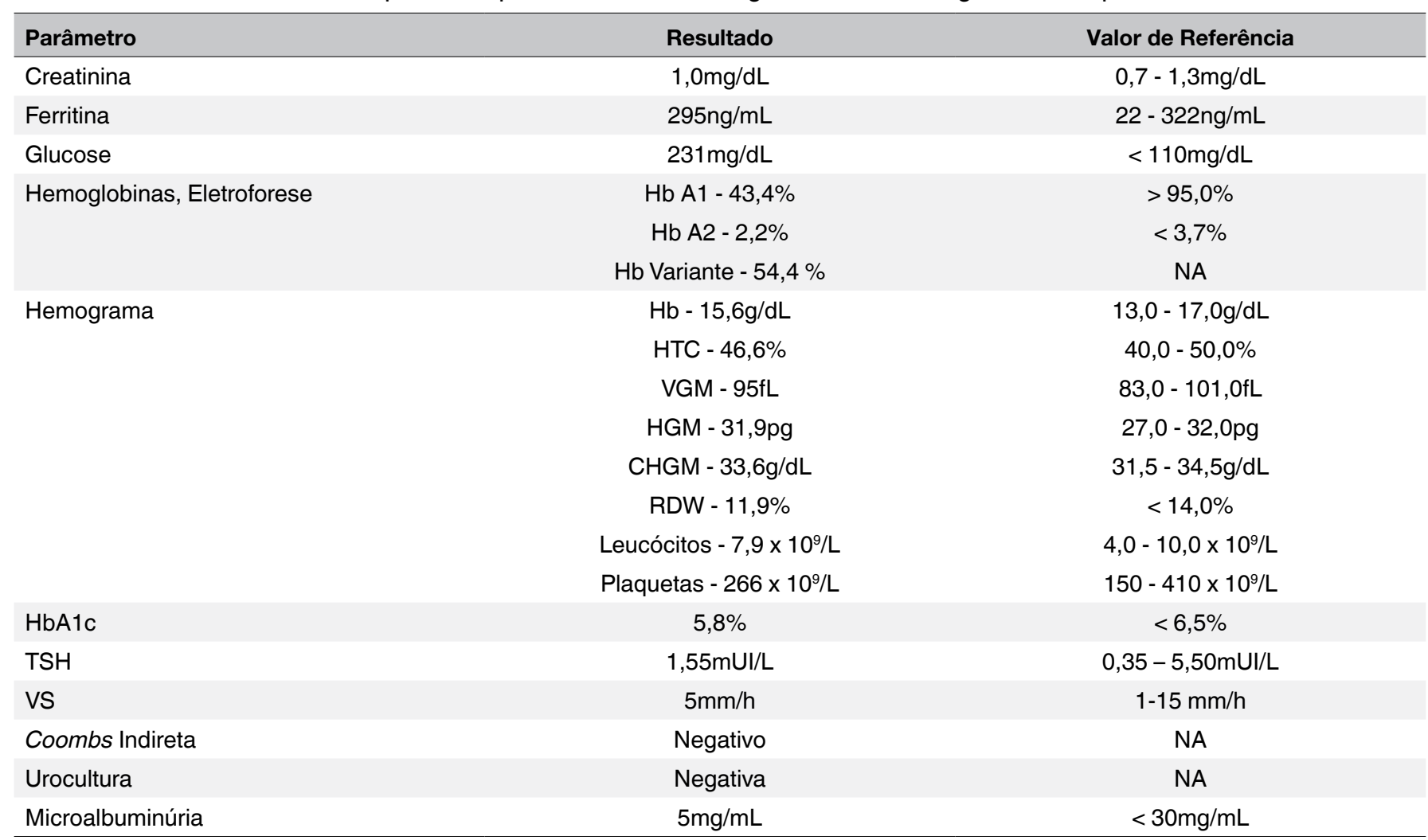

CHGM - Concentração de Hemoglobina Globular Média; Hb - Hemoglobina; HbA1c - Hemoglobina A1c; HGM - Hemoglobina Globular Média; HTC - hematócrito; NA - Não Aplicável; RDW - Red Cell Distribution Width;TSH - Thyroid-Stimulating Hormone; VGM - Volume Globular Médio; VS - Velocidade de Sedimentação. 
Perante este diagnóstico, foi feita a avaliação dos antecedentes familiares sem aspectos a valorizar. Atualmente, o paciente e a sua família estão sendo orientados em consulta de Genética Médica, mantendo o seguimento pelo seu Médico de Família.

Com um valor de $\mathrm{HbA} 1 \mathrm{c}$ de $8,4 \%$, a diabetes não apresentava um bom controle. Dada a idade, as comorbidades e a ausência de complicações micro ou macrovasculares, o nosso objetivo de HbA1c a atingir seria de 6,5-7,0\%. ${ }^{7}$ Sendo esperado que a titulação da dose de metformina apenas permitiria um decréscimo da $\mathrm{HbA} 1 \mathrm{c}$ até $1 \%$, e considerando que a profissão do doente envolve a condução automóvel com o transporte de passageiros (contraindicação para terapêuticas com risco elevado de hipoglicemia), ${ }^{8}$ foi realizado o ajuste terapêutico com titulação da metformina e associação com linagliptina $(1000 \mathrm{mg}+2,5 \mathrm{mg}$, 1 comprimido duas vezes dia). Verificou-se a redução do valor da HbA1c (HPLC conjugado com a afinidade ao boronato $\mathrm{Hb912O}$ Premier $^{\circledR}$ ) para 6,7\% após 4 meses de terapêutica.

\section{Discussão}

A HbA1c continua a ser o gold standard para monitorizar o controle glicêmico em pessoas com diabetes mellitus. ${ }^{7}$ Dois grandes ensaios clínicos prospectivos randomizados demonstraram inequivocamente que a $\mathrm{HbA1c}$ prediz o risco de complicações microvasculares a longo prazo nestes pacientes..$^{9,10}$

$\mathrm{A} \mathrm{HbA} 1 \mathrm{c}$ é formada in vivo por uma reação entre a glicose e a região $\mathrm{N}$-terminal das cadeias de $\mathrm{Hb}$ $\alpha$ ou $\beta$. A reação não enzimática irreversível entre a glicose e a $\mathrm{HbA}$, o principal tipo de $\mathrm{Hb}$ em adultos, ocorre ao longo de aproximadamente três meses (o tempo de vida do eritrócito). ${ }^{11}$ As variantes genéticas podem alterar a ligação da glicose, resultando em valores discordantes de $\mathrm{HbA} 1 \mathrm{c}$ e as medições de glicose capilar em ambulatório. ${ }^{12}$

No caso clínico apresentado, os valores de $\mathrm{HbA} 1 \mathrm{c}$ não eram compatíveis com os níveis de glicemia ao longo do dia. Existem várias situações clínicas que podem interferir no resultado do teste, promovendo a redução ou o aumento do valor real de $\mathrm{HbA1c}$, e dificultando a sua utilização adequada (Tabela 2).

Tabela 2. Condições clínicas que podem interferir no resultado do teste de $\mathrm{HbA1c}$.

Situações que podem promover HbA1c falsamente diminuída

1. Anemias hemolíticas de diferentes etiologias (ex.: algumas hemoglobinopatias)

1. Hemorragia

2. Afecção da medula óssea por radiação, toxinas, fibrose, tumores

3. Medicações (ex.: antirretrovirais, dapsona)

2. Doença renal crônica com déficit de eritropoetina

3. Gestação

Situações que podem promover $\mathrm{HbA1c}$ falsamente aumentada

1. Doença renal crônica com presença de hemoglobina carbamilada

2. Deficiência nutricional de ferro

3. Algumas hemoglobinopatias
1. Doentes que usam doses elevadas de ácido acetilsalicílico com presença de hemoglobina acetilada

2. Condições que promovem aumento do número de eritrócitos e/ou do valor do hematócrito

Após exclusão de erro na avaliação das glicemias, o paciente do caso descrito não apresentava história farmacológica (antirretrovirais, dapsona ou ácido acetilsalicílico) passível de interferência com a $\mathrm{HbA} 1 \mathrm{c}$ e dos seus antecedentes não constava exposição a radiação ou toxinas, bem como doença renal crônica ou afecções que promovam alteração do hematócrito. A realização de eletroforese das hemoglobinas estava assim indicada para exclusão de hemoglobinopatia. ${ }^{1}$ 
Bry et al. ${ }^{13}$ reviram como as hemoglobinopatias podem interferir na análise de $\mathrm{HbA} 1 \mathrm{c}$, independentemente dos efeitos na sobrevida de eritrócitos. Influência na análise da $\mathrm{HbA} 1 \mathrm{c}$ por $\mathrm{HbF}, \mathrm{HbS}, \mathrm{HbC}$ e outras formas anormais de $\mathrm{Hb}$ foi relatada. Os resultados podem ser falsamente aumentados, falsamente diminuídos ou não alterados, dependendo do método específico usado para medir a HbA1c e do tipo de hemoglobinopatia.

A Hemoglobina N-Baltimore, também conhecida como hemoglobina N-Memphis; Hopkins-I ou Kenwood, apresentada por este paciente, foi descrita por Smith e Torbert em 1958. É produzida por uma mutação na qual a lisina é substituída pelo ácido glutâmico no codão 95 da cadeia $\beta$ da molécula de hemoglobina ( $\beta 95$ Lys $\rightarrow$ Glu). Esta variante estrutural não causa nenhuma anormalidade eritrocitária e, portanto, os pacientes com essa variante de $\mathrm{Hb}$ são clinicamente assintomáticos. ${ }^{14}$

O impacto das variantes genéticas na determinação da HbA1c difere consideravelmente entre os mais de 30 métodos comercialmente disponíveis. ${ }^{15}$ Os métodos de análise da HbA1c podem ser divididos em dois grupos - ensaios baseados em carga molecular (HPLC de troca iônica e eletroforese) e em métodos que usam estrutura (imunoensaio e cromatografia de afinidade com o boronato).

Entre estes métodos de ensaio, HPLC de troca iônica (um dos mais usados) tende a demonstrar a maior interferência da presença de variantes e derivados de $\mathrm{Hb}$ nos valores de $\mathrm{HbA} 1 \mathrm{c}$. A cromatografia por afinidade do boronato, por outro lado, é um método bem documentado como sendo um dos testes de $\mathrm{HbA} 1 \mathrm{c}$ com menor interferência para indivíduos com uma variante de $\mathrm{Hb} .^{13}$

O teste da fructosamina tem por base a glicosilação de proteínas, resultando da interação entre a glicose plasmática e a lisina (presente na molécula da albumina e de outras proteínas). A dosagem da fructosamina é recomendada em situações nas quais o teste de A1c apresente algum interferente, como no caso relatado. ${ }^{1}$

Com o intuito de se evitar problemas na interpretação dos níveis de A1C obtidos pelos diversos métodos laboratoriais, foi criado um projeto especial: o National Glycohemoglobin Standardization Program (NGSP), disponível no link: http://www.ngsp.org. Este programa tem por finalidade harmonizar os métodos para dosagem da $\mathrm{HbA} 1 \mathrm{c}$, visando padronizar os resultados em relação ao método utilizado. ${ }^{15}$

Embora assintomática, o reconhecimento desta hemoglobinopatia tornou-se de vital importância para este paciente, visto que durante os primeiros 4 anos após o seu diagnóstico, a sua diabetes foi encarada falsamente como controlada, pois estávamos apenas a basear-nos nos valores de HbA1c. É questionável se, para um paciente individual, o melhor marcador para o risco de complicações será o nível de HbA1c ou o nível de glicemia média estimada. Este fato reveste-se de uma importância ainda maior em pacientes com hemoglobinopatias.

Geralmente, os médicos confiam mais nos níveis de $\mathrm{HbA} 1 \mathrm{c}$ do que na automonitorização das glicemias capilares ao tomar decisões terapêuticas, com a consequência evidente de serem feitos ajustes desadequados com implicações negativas no prognóstico e no risco de complicações dos pacientes. Como tal, em casos de persistência de inexplicável discordância entre a HbA1c e as glicemias capilares, deve ser realizada a eletroforese das hemoglobinas. ${ }^{3}$

Considerando a importância dos cuidados de proximidade do Médico de Família, foi possível realizar a abordagem correta deste paciente em Cuidados de Saúde Primários, permitindo a redução de um elevado risco de complicações decorrentes da diabetes e a melhoria do prognóstico a longo prazo deste paciente. 


\section{Referências}

1. Atualização sobre hemoglobina glicada (A1C) para avaliação do controle glicêmico e para o diagnóstico do diabetes: aspectos clínicos e laboratoriais. Posicionamento Oficial SBD, SBPC-ML, SBEM e FENAD 2017/2018 [Internet]. [acesso 2018 Set 8]. Disponível em: https:// www.diabetes.org.br/publico/images/banners/posicionamento-3-2.pdf

2. Gargallo MA, González FA, Villegas A. Abnormally low HbA1c secondary to hemoglobin J-Baltimore [beta 16(A13) Gly-->Asp]. Family study. Endocrinol Nutr. 2010;57(2):83-5. http://dx.doi.org/10.1016/j.endonu.2010.01.004

3. Beck RW, Connor CG, Mullen DM, Wesley DM, Bergenstal RM. The Fallacy of Average: How Using HbA1c Alone to Assess Glycemic Control Can Be Misleading. Diabetes Care. 2017;40(8):994-9. http://dx.doi.org/10.2337/dc17-0636

4. Wheeler E, Leong A, Liu CT, Hivert MF, Strawbridge RJ, Podmore C, et al. Impact of common genetic determinants of Hemoglobin A1c on type 2 diabetes risk and diagnosis in ancestrally diverse populations: A transethnic genome-wide meta-analysis. PLoS Med. 2017;14(9):e1002383. http://dx.doi.org/10.1371/journal.pmed.1002383

5. HbVar: A database of human hemoglobin variants and thalassemias [Internet]. [acesso 2018 Set 8]. Disponível em: http://globin.bx.psu. edu/cgi-bin/hbvar/counter

6. Bonini-Domingos CR, Silveira ELV, Viana-Baracioli LMS, Canali AA. Caracterização de hemoglobina N-Baltimore em doador de sangue de São José do Rio Preto, SP. J Bras Patol Med Lab. 2003;39(1):41-4. http://dx.doi.org/10.1590/S1676-24442003000100008

7. American Diabetes Association. 6. Glycemic Targets: Standards of Medical Care in Diabetes-2018. Diabetes Care. 2018;41(Suppl 1):S5564. http://dx.doi.org/10.2337/dc18-S006

8. Bennett WL, Maruthur NM, Singh S, Segal JB, Wilson LM, Chatterjee R, et al Comparative effectiveness and safety of medications for type 2 diabetes: an update including new drugs and 2-drug combinations. Ann Intern Med. 2011;154(9):602-13. http://dx.doi.org/10.7326/00034819-154-9-201105030-00336

9. Diabetes Control and Complications Trial Research Group, Nathan DM, Genuth S, Lachin J, Cleary P, Crofford O, Davis M, et al. The effect of intensive treatment of diabetes on the development and progression of long-term complications in insulin-dependent diabetes mellitus. N Engl J Med. 1993;329(14):977-86. http://dx.doi.org/10.1056/NEJM199309303291401

10. Intensive blood-glucose control with sulphonylureas or insulin compared with conventional treatment and risk of complications in patients with type 2 diabetes (UKPDS 33). UK Prospective Diabetes Study (UKPDS) Group. Lancet. 1998;352(9131):837-53. http://dx.doi. org/10.1016/S0140-6736(98)07019-6

11. Rahbar S. The discovery of glycated hemoglobin: a major event in the study of nonenzymatic chemistry in biological systems. Ann $\mathrm{N} Y$ Acad Sci. 2005;1043:9-19. http://dx.doi.org/10.1196/annals.1333.002

12.Sacks DB. Hemoglobin variants and hemoglobin A1c analysis: problem solved? Clin Chem. 2003;49(8):1245-7. http://dx.doi. org/10.1373/49.8.1245

13. Bry L, Chen PC, Sacks DB. Effects of hemoglobin variants and chemically modified derivatives on assays for glycohemoglobin. Clin Chem. 2001;47(2):153-63.

14. Lorenzo-Medina M, Nogueira-Salgueiro P, Martin-Aguila A, Ruiz-Garcia L. Interference of Hemoglobin N-Baltimore on Measurement of HbA1c Using the HA-8160 and HA-8180 HPLC Methods. J Diabetes Sci Technol.2015;9(3):714-5. http://dx.doi.org/10.1177/1932296815574723

15. National Glycohemoglobin Standardization Program (NGSP). Factors that interfere with HbA1c test results [Internet]. [acesso 2018 Set 7]. Disponível em: www.ngsp.org/factors.asp 